\title{
Water on clay: electrical double layer, disjoining pressure, and colloidal aggregation at Earth's most abundant interface
}

\footnotetext{
IAN C. BOURG ${ }^{1,2 *}$

${ }^{1}$ Department of Civil and Environmental Engineering, Princeton University, Princeton, New Jersey 08544, USA (*correspondence: bourg@princeton.edu)

${ }^{2}$ Princeton Environmental Institute, Princeton University, Princeton, New Jersey 08544, USA

Smectite clay minerals are natural nanoparticles that contribute predominantly to the overall mass and specific surface area of soils, sediments, and sedimentary rocks in temperate weathering environments. Here, we present recent advances on the use of molecular dynamics (MD) simulations to evaluate key aspect of the geochemistry of these minerals, notably the existence of an electrical double layer (EDL) at the clay-water interface, the role of the EDL in mediating the aggregation of smectite clay colloids, and the thermodynamic and transport properties of water within smectite clay aggregates. Our results are relevant to a range of topics including the use of clay-rich rocks in the geologic storage of $\mathrm{CO}_{2}$ and radioactive waste, the flow of water and hydrocarbons in soils and sedimentary formations, the consolidation of fine-grained sediments, and the mechanics of clay-bearing faults.
} 